\title{
Alpha-Lipoic Acid Alleviates Cerebral Ischemic Injury-Induced Cognitive Impairment and Alzheimer's Disease-Related Pathologies Through Modulation of GSK-3 $\beta$ in a Rat Model of Transient Middle Cerebral Artery Occlusion
}

Ming-Hsiu Wu ( $\nabla$ galenmhwu@yahoo.com )

Chi Mei Medical Center https://orcid.org/0000-0003-0354-2916

Chia-Yu Chang

Chi Mei Medical Center

Research Article

Keywords: dementia, vascular cognitive impairment and dementia (VCID), cerebral ischemia-reperfusion

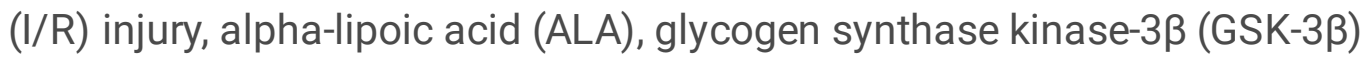

Posted Date: October 11th, 2021

DOI: https://doi.org/10.21203/rs.3.rs-960110/v1

License: (c) (1) This work is licensed under a Creative Commons Attribution 4.0 International License. Read Full License 


\section{Abstract \\ Background}

There are two main types of dementia: Alzheimer's disease (AD) and vascular cognitive impairment and dementia (VCID). Alpha-lipoic acid (ALA) has antioxidant and anti-inflammatory properties, and protects against cerebral ischemia-reperfusion (I/R) injury. We aimed to investigate the protective role of ALA in cerebral I/R injury-related cognitive impairment in a rat model of transient middle cerebral artery occlusion (tMCAO), which mimics VCID in humans.

\section{Methods}

After I/R injury, rats were treated with either an intraperitoneal injection of ALA ( $25 \mathrm{mg} / \mathrm{kg})$ or vehicle. Infarct volume, brain edema, modified neurologic severity scores, and Y-maze test for cognitive impairment were assessed. In addition, activation of glycogen synthase kinase-3 $\beta$ (GSK-3 $\beta$ ) and microglia, and expression of amyloid- $\beta$ precursor protein (APP), $\beta$-site APP cleaving enzyme 1 (BACE1), amyloid- $\beta(A \beta)$, tau, and synaptophysin were measured 7 days after tMCAO.

\section{Results}

We found that administration of ALA after cerebral I/R injury reduced cerebral infarction, brain edema, and improved neurologic deficits and cognitive impairment. In parallel, ALA reduced the expression of APP, BACE1, A $\beta$, and phosphorylated tau in the hippocampus, down-regulated the activation of GSK-3 $\beta$ and microglia, and improved the integrity of neuronal synapses.

\section{Conclusion}

ALA alleviated the severity of cerebral I/R injury and AD-related pathologies, preserved neuronal integrity, and improved cognitive impairment by down-regulating the activation of GSK-3 $\beta$ and microglia.

\section{Background}

Dementia is a clinically challenging disease worldwide as we face an aging society [1]. Vascular cognitive impairment and dementia (VCID) is a major subtype of dementia resulting from a spectrum of cerebral vascular diseases [2], while Alzheimer's disease (AD) is a major subtype of dementia due to neurodegeneration $[3,4]$. Although the importance of VCID has received growing attention, the mechanisms related to the neuropathological changes that result in cognitive decline are complex, and effective treatment is still lacking [2]. It has been shown that cerebral ischemia could lead to AD-related pathologies [5]. 
Glycogen synthase kinase (GSK), a constitutive protein kinase, is not only involved in diverse physiological functions, but also in the pathogenesis of many diseases [6]. GSK-3 $\beta$ activation has been found in cerebral ischemic injury [7-9]. Inhibition of GSK-3 $\beta$ activation has been shown to protect against ischemic neuronal injury. In addition, GSK-3 $\beta$ has been shown to play a detrimental role in the pathogenesis of $A D$ [10]. Its activation has been linked to $A \beta$ production and hyperphosphorylation of tau.

Alpha-lipoic acid (ALA) is a compound that through multiple mechanisms protects against cerebral ischemic injury, including anti-oxidant and anti-inflammatory activities [11]. However, whether it is beneficial in reducing cerebral I/R injury-related cognitive impairment and AD-related pathologies is not clear. The purpose of this study was to test the hypothesis that ALA protects against cognitive impairment and AD-related pathologies after cerebral I/R injury in a rat model of transient middle cerebral artery occlusion (tMCAO) through modulation of GSK-3ß.

\section{Materials And Methods}

\section{Animals and Treatments}

Adult, male Sprague-Dawley rats (280 to $320 \mathrm{~g}$, BioLASCO Taiwan Co., Ltd., Taipei, Taiwan) were used in this study. The rats were housed in groups of 4 at an ambient temperature of $22 \pm 1^{\circ} \mathrm{C}$ with a 12-h lightdark cycle according to the guidelines of the Institutional Animal Care and Use Committee at Chi Mei Medical Center (approval no. 104122916). Pellet rat chow and tap water were available ad libitum. The rats were anesthetized with Rompun and Zoletil 50 (Virbac of Brazil, Brazil) $(0.01$ and $0.04 \mathrm{mg} / \mathrm{g}$ of body weight, respectively). ALA (purity of $>99 \%$ ) was purchased from Sigma Biotechnology (St. Louis, MO, USA) and dissolved in $10 \%$ ethanol. Rats were randomly assigned to three experimental groups: sham, MCAO + vehicle ( $1 \mathrm{mg} / \mathrm{kg})$, and MCAO + ALA $(25 \mathrm{mg} / \mathrm{kg})$. ALA and vehicle ( $10 \%$ ethanol) were administered intraperitoneally 120 minutes after the onset of cerebral ischemia.

\section{tMCAO Model of Rats}

tMCAO was induced using the intraluminal filament procedure of Koizumi et al. and Longa et al. [12,13]. A midline cervical incision was made, and the right common carotid artery (CCA), external carotid artery (ECA), and internal carotid artery (ICA) were exposed. A length of 4-0 surgical nylon suture (20 to $21 \mathrm{~mm}$ ) with a silicon rubber-coated head of $0.4 \mathrm{~mm}$ diameter (403956PK10; Doccol) [14] was inserted from the ECA into the lumen of the ICA to block the flow of middle cerebral artery (MCA). One hundred and twenty minutes after the completion of MCA occlusion, reperfusion was allowed by withdrawal of the suture thread until the tip cleared the ICA. Sham-operated animals underwent the same surgical procedure, in which suture was inserted without silicon rubber-coated head into the ICA.

\section{Behavioral Evaluation of Neurological Deficits - Modified Neurologic Severity Score (mNSS)}

Modified neurologic severity scores (mNSS) were used by an investigator who was blinded to the experimental groups to assess the severity of neurological deficits in rats after TMCAO. The mNSS was 
evaluated as previously described [15]. The mNSS consists of motor, sensory, balance, and reflex tests, and is graded from 0 to 18 (normal score 0 ; maximal deficit score 18) to evaluate the severity of injury.

\section{Y Maze Test}

The Y-maze test $[16,17]$ was used to evaluate cognitive impairment in rats after tMCAO. The Y-shaped maze is composed of three white and opaque plastic arms ( $35 \mathrm{~cm}$ long, $25 \mathrm{~cm}$ high, and $10 \mathrm{~cm}$ wide) equally spaced at a $120^{\circ}$ angle from each other. The rats were allowed to freely explore the three arms for 8 minutes. The rat movements inside the arms were recorded by a digital CCD camera mounted above the apparatus and were analyzed using the Top Scan system software (ver. 3.00; Clever Sys., Inc., VA, USA). The spontaneous alternation percentage (SA\%) was calculated as the actual alternations/maximum alternations $\times 100$.

\section{The Measurements of Infarct Volumes and Brain Edema}

Seventy-two hours after tMCAO, rats ( $\mathrm{n}=8$ for each group) were anesthetized and decapitated. Their brains were rapidly removed and sliced into six 2-mm-thick coronal sections. These slices were incubated in a solution of $2 \% 2,3,5$-triphenyltetrazolium chloride (TTC, Sigma) in a water bath in the dark at $37^{\circ} \mathrm{C}$ for 30 minutes, and were then photographed separately using a digital camera (Canon, Japan). Infarct was defined as unstained areas. The total infarct volume was measured in each slice and summed using computerized planimetry, as previously described $[18,19]$. For brain edema measurement, the percentage was calculated using the following formula : \% brain edema $=$ (the volume of ischemic hemisphere-the volume of the contralateral hemisphere)/the volume of the contralateral hemisphere) $\times 100$.

\section{Immunofluorescence Assay}

Seven days after cerebral ischemia-reperfusion injury, rats were perfused transcardially with $0.9 \% \mathrm{NaCl}$. The brains were removed from the skulls and post-fixed in 10\% formaldehyde. Brain tissues were sectioned at a thickness of $4 \mu \mathrm{m}$ according to the standard procedure. The sections were deparaffinized and hydrated gradually, as examined by immunofluorescence. Briefly, tissue sections were soaked in 0.01 $\mathrm{M}$ citrate buffer ( $\mathrm{pH} 6.0)$, heated to the boiling point, blocked with $10 \%$ normal goat serum for 30 minutes at $37^{\circ} \mathrm{C}$, and then incubated overnight at $4^{\circ} \mathrm{C}$ with mouse monoclonal antibody to activate microglia marker lonized calcium-binding adaptor molecule 1 (Iba1) (1:200, Abcam), rabbit monoclonal antibody to synaptophysin antibody (1:200, Abcam), rabbit monoclonal antibody to amyloid precursor protein (1:200, Abcam), mouse monoclonal antibody to GSK3 beta (1:200, Abcam), rabbit monoclonal antibody to BACE1 (1:200, Abcam), rabbit polyclonal antibody to beta Amyloid 1-42 (1:200, Abcam), and rabbit monoclonal antibody to Tau (phospho S396) (1:200, Abcam). Following washing in PBS ( $3 \times 5$ minutes), sections were incubated with goat anti-rabbit IgG H\&L (DyLight ${ }^{\circledR}$ 594) (1:200, Abcam), goat anti-mouse

IgG H\&L (DyLight $\left.{ }^{\circledR} 488\right)$ (1:200, Abcam), goat anti-mouse IgG H\&L (DyLight® ${ }^{\circledR}$ 594) (1:200, Abcam), and goat anti-rabbit IgG H\&L (DyLight@ $\left.{ }^{\circledR} 488\right)\left(1: 200\right.$, Abcam) for 50 minutes at 25 $5^{\circ}$ C. 4,6-Diamidino-2phenylindole (DAPI; 1:5000; Invitrogen) was used to identify nuclear DNA. After mounting with glycerol gelatin (Sigma), the slides were examined under epi-fluorescence on an Olympus BX60 microscope. 


\section{Statistical Analysis}

All data are expressed as the mean \pm standard error of the mean (SEM). Statistical comparisons were performed using the Kruskal-Wallis test, followed by post hoc comparisons. The Dunn method was used to compare more than two groups, while the Mann-Whitney $\mathrm{U}$ test was used for comparing two groups. $\mathrm{P}$ values less than 0.05 were considered as statistically significant differences.

\section{Results}

\section{ALA alleviated severity of cerebral I/R injury and related neurologic deficits and cognitive impairment}

To investigate whether ALA can alleviate the severity of cerebral I/R injury, we measured the area of TTC stain and brain edema at 3 days, and neurobehavioral deficits 3 and 7 days after tMCAO. We found that ALA reduced the infarct area and brain edema (Fig. 1A, 1B, and 1C). ALA improved neurological deficits as measured by mNSS 3 and 7 days after MCAO (Fig. 1D).

To investigate whether ALA can alleviate cerebral I/R injury-related cognitive impairment, we measured the spontaneous alternation percentage in $Y$ maze test 3,7 , and 28 days after $\mathrm{TMCAO}$. As shown in Fig. $1 \mathrm{E}$, rats receiving ALA showed a higher spontaneous alternation percentage in the $\mathrm{Y}$-maze test than those receiving vehicle.

\section{ALA alleviated activation of GSK-3 $\beta$ and microglia after cerebral I/R injury}

To evaluate GSK-3 $\beta$ activation after cerebral I/R injury, we measured the levels of phosphorylated GSK-3 $\beta$ by immunofluorescence staining in the rat hippocampus 7 days after TMCAO. ALA was found to significantly alleviate the activation of GSK-3 3 after cerebral I/R injury (Fig. 2A, 2B). To assess the activation of microglia after cerebral I/R injury, we examined the levels of activated microglia marker Iba1 with immunofluorescence staining in the rat hippocampus 7 days after tMCAO. Furthermore, ALA significantly alleviated the activation of microglia after cerebral I/R injury (Fig. 2C, 2D).

\section{ALA alleviated AD-related pathologies and maintained neuronal integrity after cerebral l/R injury}

To characterize the pattern of AD-related pathologies after cerebral I/R injury, we measured the expression of $A P P, B A C E 1, A B$, and tau in the rat hippocampus using immunofluorescence staining 7 days after tMCAO. As shown in figure 3 , the expression of the above-mentioned enzyme and proteins increased significantly in the rat hippocampus after cerebral I/R injury, which was significantly alleviated by the administration of ALA.

To evaluate the neuronal integrity after cerebral I/R injury, we measured the expression of synaptophysin in the rat hippocampus using immunofluorescence staining 7 days after TMCAO. As shown in figure 4, 
ALA maintained neuronal integrity by increasing the number of neuronal synapses and improving their alignment in the rat hippocampus after cerebral I/R injury.

\section{Discussion}

In this study, we demonstrated that ALA alleviated cognitive impairment and AD-related pathologies after cerebral ischemia-reperfusion injury in a rat model of tMCAO through down-regulation of GSK-3 $\beta$. After transient cerebral ischemia for $2 \mathrm{~h}$, cerebral infarction and brain edema were found, and consequent neurologic deficits and cognitive impairment were observed in tMCAO rats. Meanwhile, GSK-3 $\beta$ and microglia were activated in the hippocampus. Furthermore, increased expression of AD-related proteins (APP, BACE1, A 3 , and tau) and reduced expression of synaptophysin were found in the hippocampus 7 days after cerebral I/R injury. It was found that administration of ALA after cerebral I/R injury reduced cerebral infarction, brain edema, and improved neurologic deficits and cognitive impairment. In parallel with these findings, ALA reduced the expression of APP, BACE1, A 3 , and phosphorylated tau in the hippocampus, down-regulated the activation of GSK-3 $\beta$ and microglia, and improved the integrity of neuronal synapses.

To the best of our knowledge, this is the first report indicating that ALA can down-regulate the activation of GSK-3 $\beta$ after cerebral I/R injury and reduce AD-related pathologies, as we demonstrated in this study. It has been shown that cerebral hypoxia-ischemia can increase the production of APP and activate BACE1, and further increase the production of $A B[5]$ and the levels of Tau proteins $[5,20]$, suggesting that cerebral ischemia can produce AD-related pathologies, including activation of BACE1 and increased production of APP, $A \beta$, and Tau. Indeed, our findings support this notion (Fig. 3).

ALA is an organosulfur compound displaying mainly anti-oxidant and anti-inflammatory activity [21, 22]. The application of ALA in diverse diseases, including CNS diseases, has been receiving growing attention, [23-25]. Among them, ALA has long been recognized to be protective against cerebral I/R injury [25]. The mechanisms through which ALA protects against cerebral I/R injury involve mainly its anti-oxidant and anti-inflammatory activities. In the current study, we demonstrated the anti-inflammatory protective effects of ALA against the activation of GSK-3 $\beta$ and microglia that may lead to cognitive impairment and AD-related pathologies after cerebral I/R injury. In fact, activation of GSK-3 $\beta$ and microglia play an important role in the pathological consequences of cerebral I/R injury.

GSK is a constitutive protein kinase, with multiple function involving diverse pathophysiological pathways. Physiologically, it has been found to be involved in the regulation of cell cycle, gene expression, development, and metabolism [26]. Pathologically, it is involved in psychiatric diseases, neurological diseases, cardiovascular diseases, diabetes mellitus, inflammatory diseases, bone diseases, and cancer [6]. GSK-3 $\beta$ activation has been found in cerebral ischemic injury [7-9]. Application of GSK-3 $\beta$ inhibitors protects against ischemic neuronal injury. GSK-3 $\beta$ activation has been noted in the pathophysiological processes leading to $A D[10,27]$. Moreover, GSK-3 $\beta$ activation seems to be involved in the production of AD-related pathologies after cerebral ischemic injury. GSK-3 $\beta$ activation has been 
correlated with increased production of APP $[28,29]$ and hyperphosphorylation of Tau [28] in the neonatal rat brain after hypoxia-ischemia. Furthermore, inhibition of GSK-3 $\beta$ decreased the production of APP [28] and hyperphosphorylation of Tau $[28,30]$, and exerted its neuroprotective activity against cerebral I/R injury. In the current study, we clearly demonstrated that reduction in the activation of GSK-3 $\beta$ by ALA reduced $A D$-related pathologies, including activation of BACE1 and production of $A P P, A B$, and Tau in cerebral I/R injury (Fig. 3).

Although not directly connected to cerebral ischemia, GSK-3 $\beta$ activation has been shown to lead to microglial activation in another inflammatory condition. Green and Nolan [31] have shown that lipopolysaccharide (LPS) can induce an increase in GSK-3 $\beta$ expression and activity in microglia in vitro. The current study showed that GSK-3 $\beta$ activation correlated well with microglia activation, since ALA down-regulated GSK-3 $\beta$ activation in parallel with microglia activation in cerebral I/R injury.

Microglial activation has been noted for a long time in cerebral ischemic injury [32]. The early activation of microglia in cerebral ischemic injury is detrimental to the brain. In our previous study, it was found that early administration of ALA can alleviate cerebral I/R injury in rats by down-regulating microglia activation [11]. In the current study, early administration of ALA not only alleviated cerebral I/R injury but also improved cognitive impairment and reduced AD-related pathologies through down-regulation of microglia activation.

Nevertheless, this is the first study indicating that ALA can down-regulate the expression of GSK-3 $\beta$ after cerebral I/R injury. Furthermore, this is also the first study indicating that ALA can reduce AD-related pathologies after cerebral I/R injury. In the current study, ALA was shown to down-regulate the activation of GSK-3 $\beta$ and microglia, and reduce AD-related pathologies, including the expression of APP, BACE1, A $\beta$, and tau after cerebral I/R injury.

\section{Limitations}

Our study is a descriptive study. It demonstrated the cascade of Alzheimer's disease-related neuropathological changes after cerebral ischemic injury in the rat model of transient middle cerebral artery occlusion. Future work is needed in mechanistic exploration regarding how ALA regulates GSK-3 $\beta$ after cerebral ischemic injury. Besides, this is a in vivo study and it warrants further efforts in future application of ALA in clinical treatment. In fact, ALA has been used as either the nutritional supplement or the drug in the treatment of diabetic polyneuropathy in many countries. Hence, the translational use of ALA in the treatment of VCID can be expected at least in terms of safety issues.

\section{Conclusion}

In summary, in addition to its role in AD pathology, GSK-3 $\beta$ might also be involved in VCID, which also shows AD-related pathologies after cerebral I/R injury. Importantly, AD and VCID account for the majority of dementia cases worldwide. In this study, we demonstrated that ALA can not only reduce the severity of cerebral infarction, brain edema, and neurobehavioral deficits, but also of AD-related pathologies, 
preserved the integrity of neuronal synapses, and improved cognitive impairment after cerebral I/R injury. Our study addresses the potential of ALA in the treatment of VCID after acute ischemic stroke in future clinical practice.

\section{Abbreviations}

$A D$, Alzheimer's disease; $A \beta$, amyloid- $\beta$; ALA, alpha-lipoic acid; APP, amyloid- $\beta$ precursor protein; BACE-1, $\beta$ -

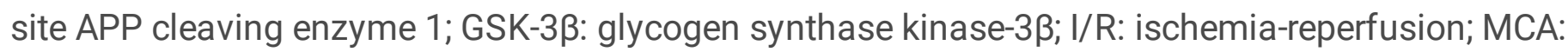
middle cerebral artery; mNSS: modified neurologic severity scores; tMCAO: transient middle cerebral artery occlusion; TTC: triphenyltetrazolium chloride; VCID: vascular cognitive impairment and dementia

\section{Declarations}

\section{Acknowledgements}

The author is grateful to Prof. Chun-Y. Hsu for mentoring, and colleagues of Chi Mei Medical Center, Liouying for support.

\section{Authors' contributions}

M.H.W. was responsible for study conceptualization and design , drafting and revising the manuscript, and obtaining funding. C.Y.C was responsible for study conceptualization and revising the manuscript.

\section{Funding}

This study was supported by Chi Mei Medical Center, Liouying (No. CLFHR10715).

\section{Consent for publication}

Not applicable.

\section{Availability of data and materials}

All data and material present in this study will be available upon reasonable request to the corresponding author.

\section{Competing interests}

The authors declare that they have no competing interests.

\section{Ethics approval and consent to participate}

This study was approved by the Institutional Animal Care and Use Committee of Chi Mei Medical Center (approval no. 104122916). 


\section{Authors' information}

${ }^{1}$ Division of Neurology, Department of Internal Medicine, Chi Mei Medical Center, Liouying, Tainan, Taiwan; ${ }^{2}$ Department of Long-Term Care and Health Promotion, Min-Hwei Junior College of Health Care Management, Tainan, Taiwan; ${ }^{3}$ Department of Neurology, Chi Mei Medical Center, Tainan, Taiwan; ${ }^{4}$ Center for General Education, Southern Taiwan University of Science and Technology, Tainan, Taiwan.

\section{References}

1. Wu YT, Beiser AS, Breteler MMB, Fratiglioni L, Helmer C, Hendrie HC, et al. The changing prevalence and incidence of dementia over time - current evidence. Nature reviews Neurology. 2017; 13(6):32739.

2. O'Brien JT, Thomas A. Vascular dementia. Lancet (London, England). 2015;386 (10004):1698-706.

3. Ballard C, Gauthier S, Corbett A, Brayne C, Aarsland D, Jones E. Alzheimer's disease. Lancet (London, England). 2011; 377(9770):1019-31.

4. Querfurth HW, LaFerla FM. Alzheimer's disease. The New England journal of medicine. 2010; 362(4):329-44.

5. Ulamek-Koziol M, Pluta R, Bogucka-Kocka A, Januszewski S, Kocki J, Czuczwar SJ. Brain ischemia with Alzheimer phenotype dysregulates Alzheimer's disease-related proteins. Pharmacological reports : PR. 2016;68(3):582-91.

6. Beurel E, Grieco SF, Jope RS. Glycogen synthase kinase-3 (GSK3): regulation, actions, and diseases. Pharmacology \& therapeutics. 2015; 148:114-31.

7. Rana AK, Singh D. Targeting glycogen synthase kinase-3 for oxidative stress and neuroinflammation: Opportunities, challenges and future directions for cerebral stroke management. Neuropharmacology. 2018; 139:124-36.

8. Hanumanthappa P, Densi A, Krishnamurthy RG. Glycogen synthase kinase-beta3 in ischemic neuronal death. Current neurovascular research. 2014;11(3):271-8.

9. Chuang DM, Wang Z, Chiu CT. GSK-3 as a Target for Lithium-Induced Neuroprotection Against Excitotoxicity in Neuronal Cultures and Animal Models of Ischemic Stroke. Frontiers in molecular neuroscience. 2011;4:15.

10. Llorens-Martin M, Jurado J, Hernandez F, Avila J. GSK-3beta, a pivotal kinase in Alzheimer disease. Frontiers in molecular neuroscience. 2014;7:46.

11. Wu MH, Huang CC, Chio CC, Tsai KJ, Chang CP, Lin NK, et al. Inhibition of Peripheral TNF-alpha and Downregulation of Microglial Activation by Alpha-Lipoic Acid and Etanercept Protect Rat Brain Against Ischemic Stroke. Molecular neurobiology. 2016;53(7):4961-71.

12. Takano K, Tatlisumak T, Bergmann AG, Gibson DG, 3rd, Fisher M. Reproducibility and reliability of middle cerebral artery occlusion using a silicone-coated suture (Koizumi) in rats. Journal of the neurological sciences. 1997;153(1):8-11. 
13. Longa EZ, Weinstein PR, Carlson S, Cummins R. Reversible middle cerebral artery occlusion without craniectomy in rats. Stroke. 1989;20(1):84-91.

14. Ishizaka S, Horie N, Satoh K, Fukuda Y, Nishida N, Nagata I. Intra-arterial cell transplantation provides timing-dependent cell distribution and functional recovery after stroke. Stroke. 2013;44(3):720-6.

15. Chen J, Sanberg PR, Li Y, Wang L, Lu M, Willing AE, et al. Intravenous administration of human umbilical cord blood reduces behavioral deficits after stroke in rats. Stroke. 2001;32(11):2682-8.

16. Wahl F, Allix M, Plotkine M, Boulu RG. Neurological and behavioral outcomes of focal cerebral ischemia in rats. Stroke. 1992;23(2):267-72.

17. Dellu F, Mayo W, Cherkaoui J, Le Moal M, Simon H. A two-trial memory task with automated recording: study in young and aged rats. Brain research. 1992;588(1):132-9.

18. Swanson RA, Morton MT, Tsao-Wu G, Savalos RA, Davidson C, Sharp FR. A semiautomated method for measuring brain infarct volume. Journal of cerebral blood flow and metabolism : official journal of the International Society of Cerebral Blood Flow and Metabolism. 1990;10(2):290-3.

19. Gartshore G, Patterson J, Macrae IM. Influence of ischemia and reperfusion on the course of brain tissue swelling and blood-brain barrier permeability in a rodent model of transient focal cerebral ischemia. Experimental neurology. 1997;147(2):353-60.

20. Zheng GQ, Wang $X M$, Wang $Y$, Wang $X T$. Tau as a potential novel therapeutic target in ischemic stroke. Journal of cellular biochemistry. 2010;109(1):26-9.

21. Moura FA, de Andrade KQ, dos Santos JC, Goulart MO. Lipoic Acid: its antioxidant and antiinflammatory role and clinical applications. Curr Top Med Chem. 2015;15(5):458-83.

22. Tibullo D, Li Volti G, Giallongo C, Grasso S, Tomassoni D, Anfuso CD, et al. Biochemical and clinical relevance of alpha lipoic acid: antioxidant and anti-inflammatory activity, molecular pathways and therapeutic potential. Inflamm Res. 2017;66(11):947-59.

23. Salehi B, Berkay Yılmaz Y, Antika G, Boyunegmez Tumer T, Fawzi Mahomoodally M, Lobine D, et al. Insights on the Use of a-Lipoic Acid for Therapeutic Purposes. Biomolecules. 2019;9(8).

24. de Sousa CNS, da Silva Leite CMG, da Silva Medeiros I, Vasconcelos LC, Cabral LM, Patrocínio CFV, et al. Alpha-lipoic acid in the treatment of psychiatric and neurological disorders: a systematic review. Metab Brain Dis. 2019;34(1):39-52.

25. Seifar F, Khalili M, Khaledyan H, Amiri Moghadam S, Izadi A, Azimi A, et al. a-Lipoic acid, functional fatty acid, as a novel therapeutic alternative for central nervous system diseases: A review. Nutr Neurosci. 2019;22(5):306-16.

26. Rayasam GV, Tulasi VK, Sodhi R, Davis JA, Ray A. Glycogen synthase kinase 3: more than a namesake. British journal of pharmacology. 2009;156(6):885-98.

27. Maqbool M, Mobashir M, Hoda N. Pivotal role of glycogen synthase kinase-3: A therapeutic target for Alzheimer's disease. European journal of medicinal chemistry. 2016;107:63-81.

28. Xiong T, Qu Y, Wang H, Chen H, Zhu J, Zhao F, et al. GSK-3beta/mTORC1 Couples Synaptogenesis and Axonal Repair to Reduce Hypoxia Ischemia-Mediated Brain Injury in Neonatal Rats. Journal of 
neuropathology and experimental neurology. 2018;77(5):383-94.

29. Xiong T, Tang J, Zhao J, Chen H, Zhao F, Li J, et al. Involvement of the Akt/GSK-3beta/CRMP-2 pathway in axonal injury after hypoxic-ischemic brain damage in neonatal rat. Neuroscience. 2012;216:123-32.

30. Cardona-Gomez GP, Arango-Davila C, Gallego-Gomez JC, Barrera-Ocampo A, Pimienta H, GarciaSegura LM. Estrogen dissociates Tau and alpha-amino-3-hydroxy-5-methylisoxazole-4-propionic acid receptor subunit in postischemic hippocampus. Neuroreport. 2006;17(12):1337-41.

31. Green HF, Nolan YM. GSK-3 mediates the release of IL-1 $\beta$, TNF- $a$ and IL-10 from cortical glia. Neurochem Int. 2012;61(5):666-71.

32. Guruswamy R, ElAli A. Complex Roles of Microglial Cells in Ischemic Stroke Pathobiology: New Insights and Future Directions. International journal of molecular sciences. 2017;18(3).

\section{Figures}



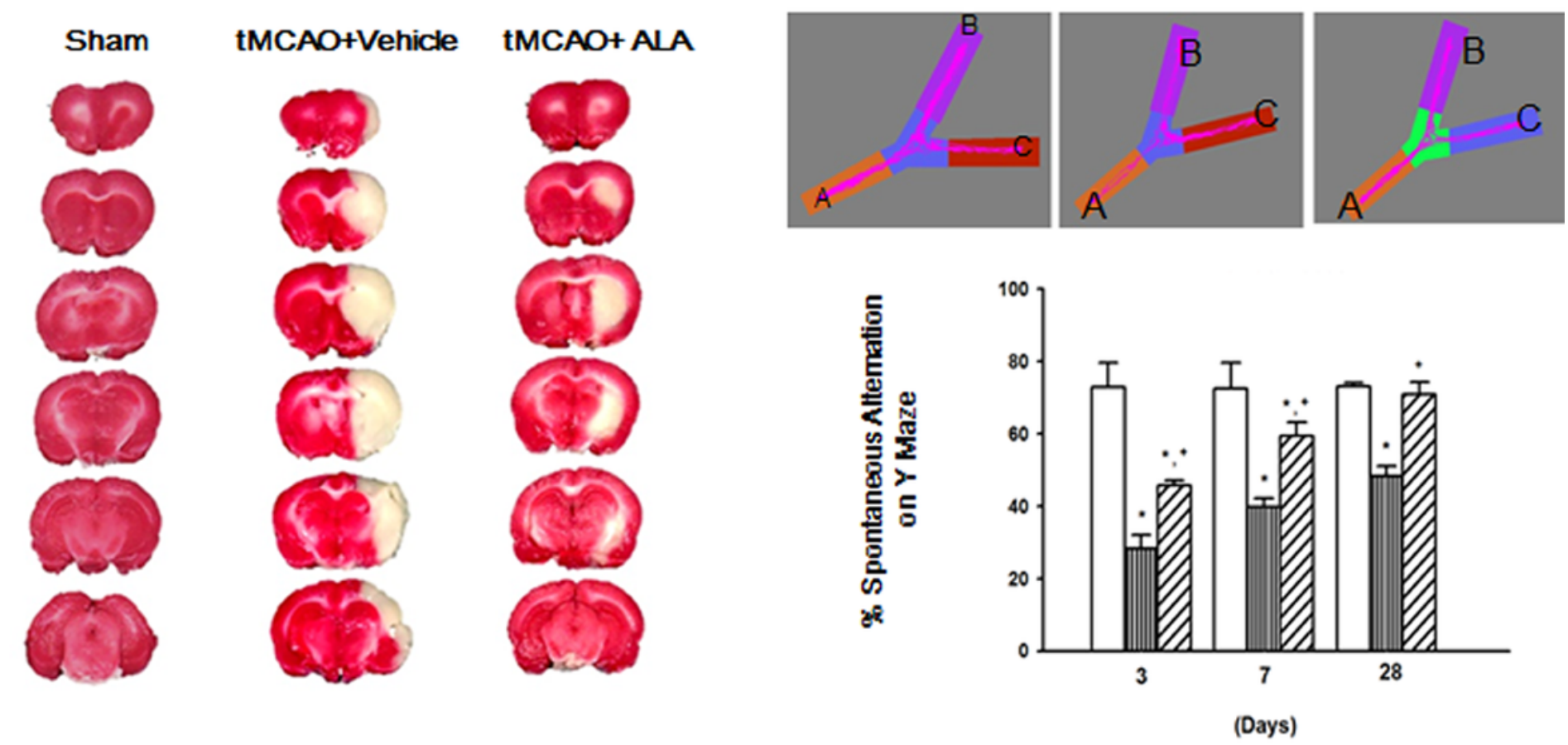

(B)

(C)


Figure 1

Alpha-lipoic acid (ALA) reduced stroke severity and cognitive impairment after cerebral ischemiareperfusion (I/R) injury. (A) representative photographs of coronal brain sections stained with triphenyltetrazolium chloride (TTC) and quantification of infarct volume (B) and brain edema (C) in sham, and vehicle or ALA treated rats 3 days after transient middle cerebral artery occlusion (tMCAO) ( $n=8$ for each group). (D) mNSS scores in the sham, and vehicle or ALA treated rats 3 days after TMCAO ( $n=8$ for each group). (E) spontaneous alternation percentage in $Y$ maze test in the sham, and vehicle or ALA treated rats 3,7 , and 28 days after $\mathrm{tMCAO}$ ( $\mathrm{n}=8$ for each group). Data are presented as the mean $\pm S D$. $* P<0.05, A L A /$ control vehicle group vs. the sham group; $+P<0.05$, the ALA group vs. the control vehicle group 
(A)

(B)

\section{GSK-3 $\beta$ stain}
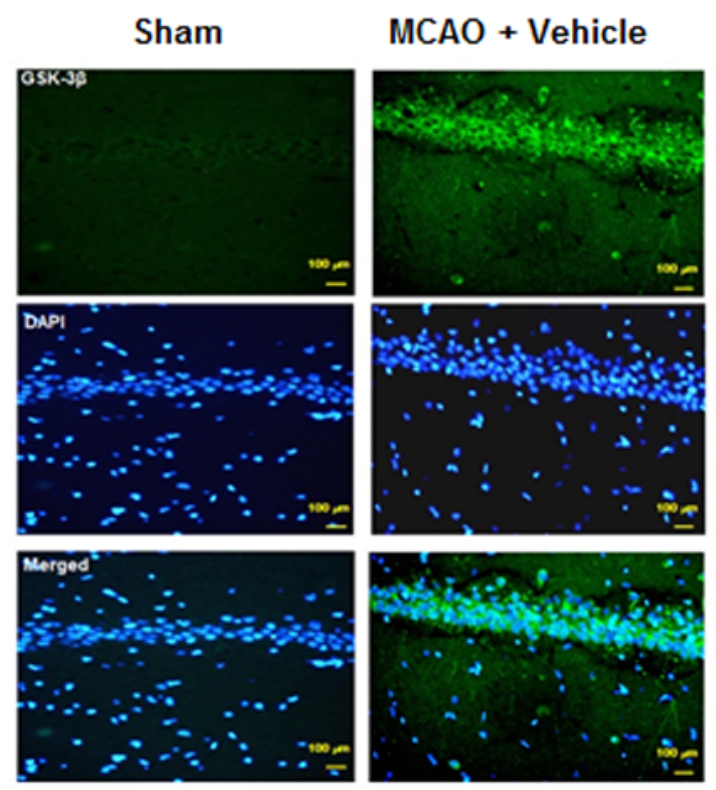

MCAO + ALA

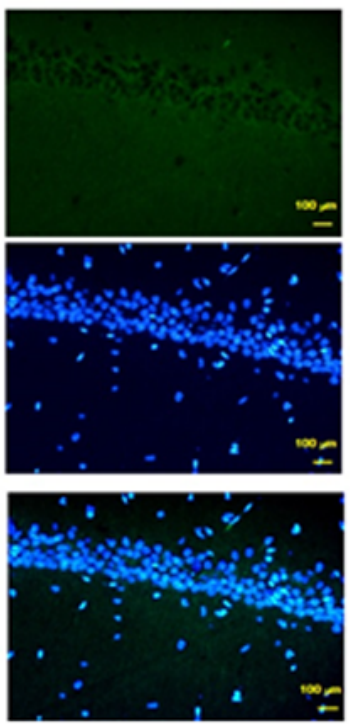

$$
\begin{aligned}
& \square \text { Sham } \\
& \square \text { tMCAO+Vehick } \\
& \square \text { tMCAO+ ALA }
\end{aligned}
$$



(C)

Microglia- Iba1 stain

(D)

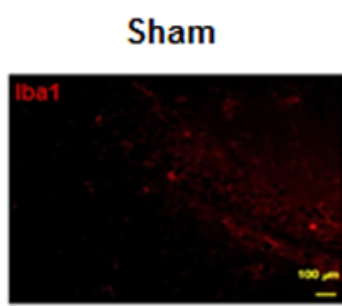

MCAO + Vehicle

MCAO + ALA
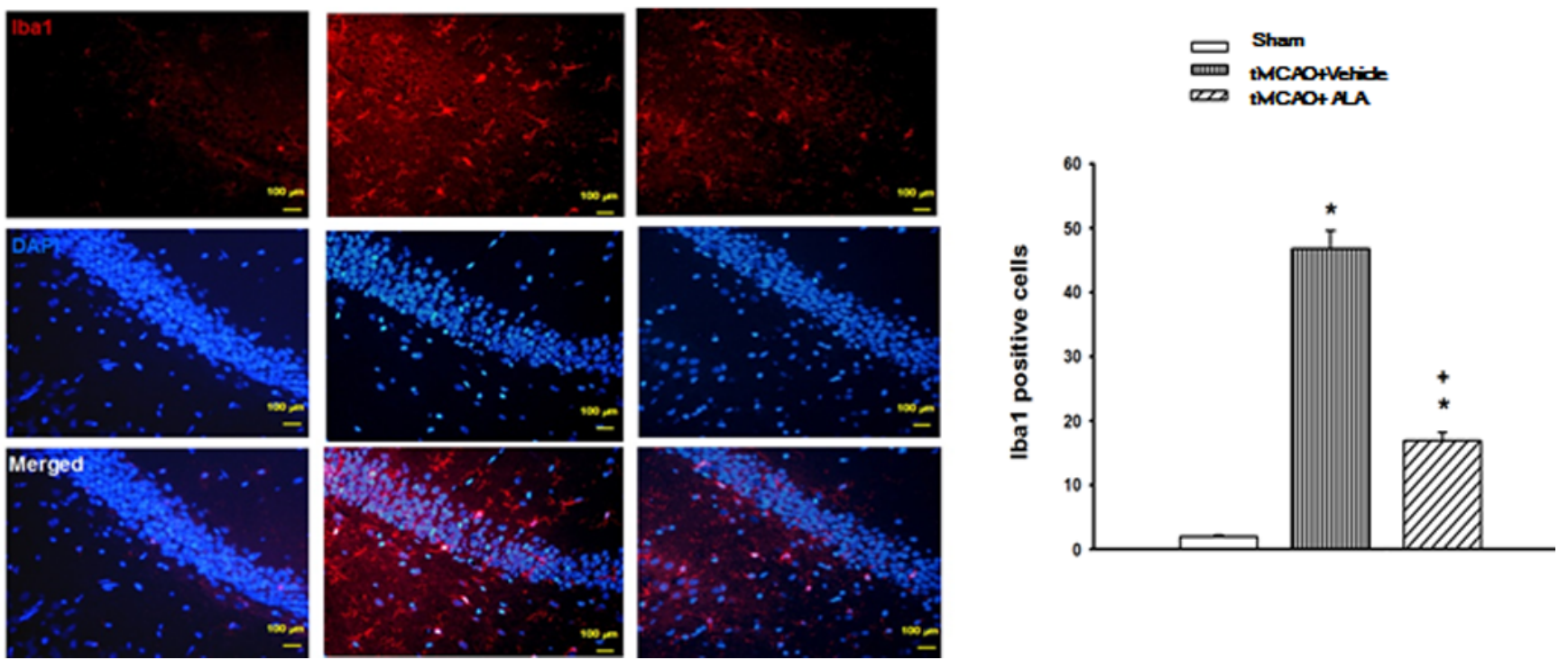

Figure 2

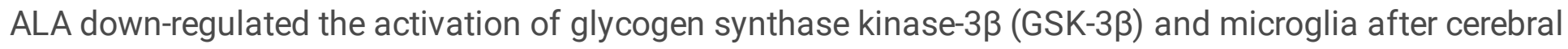
I/R injury. (A) representative photographs of coronal hippocampal sections stained with GSK-3 $\beta$ and (B) quantification of expression of GSK-3 $\beta$ in sham, and vehicle or ALA treated rats 7 days after $\mathrm{AMCAO}(\mathrm{n}=8$ for each group). (C) representative photographs of coronal hippocampal sections stained with Iba1 and (D) quantification of expression of activated microglia in the sham, and vehicle or ALA treated rats 7 days 
after $\mathrm{tMCAO}$ ( $\mathrm{n}=8$ for each group). Data are presented as the mean $\pm S D$. ${ }^{*}<0.05, A L A /$ control vehicle group vs. the sham group; $+P<0.05$, the ALA group vs. the control vehicle group

(A)

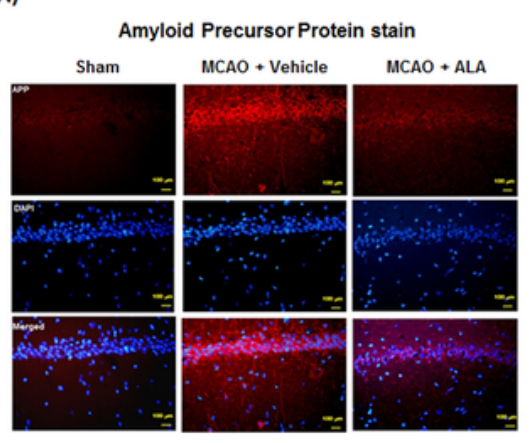

(c)

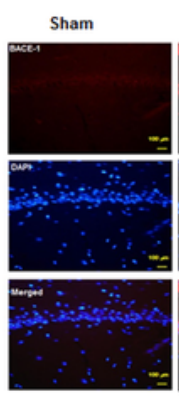

BACE-1 stain

MCAO + Vehicle
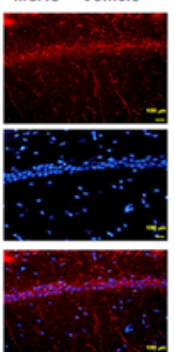

(B)

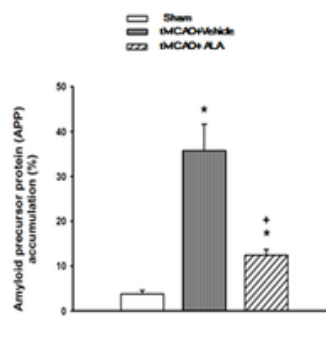

(D)

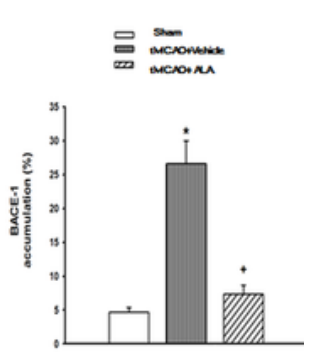

(E)

(F)

beta Amyloid stain (7D) (hippocampus)



㤩 secroments



(G) Tau (phospho \$396) stain (7D) (hippocampus)

(H)
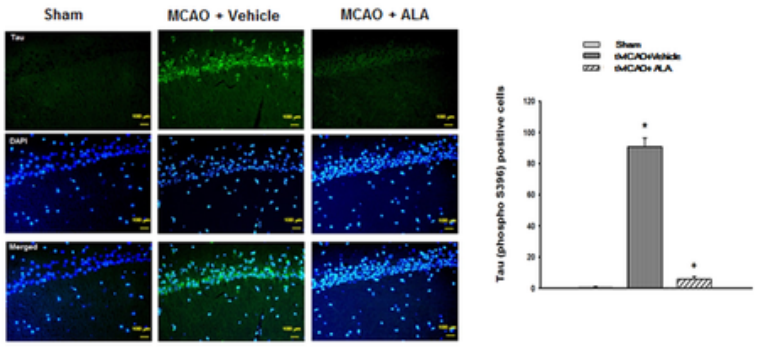

\section{Figure 3}

ALA reduced the expression of amyloid- $\beta$ precursor protein (APP), $\beta$-site APP cleaving enzyme 1 (BACE-1), amyloid- $\beta$ (AB), and Tau after cerebral I/R injury. (A) representative photographs of coronal hippocampal sections stained for APP and (B) quantification of expression of APP in sham, and vehicle or ALA treated 
rats 7 days after tMCAO ( $n=8$ for each group). (C) representative photographs of coronal hippocampal sections stained with BACE-1 and (D) quantification of expression of BACE-1 in sham, and vehicle or ALA treated rats 7 days after $\mathrm{tMCAO}$ ( $n=8$ for each group). (E) representative photographs of coronal hippocampal sections stained with $A \beta$ and $(F)$ quantification of the expression of $A \beta$ in sham, and vehicle or ALA treated rats 7 days after tMCAO ( $n=8$ for each group). ( $G$ ) representative photographs of coronal hippocampal sections stained for Tau and $(\mathrm{H})$ quantification of the expression of Tau protein in sham, and vehicle or ALA treated rats 7 days after $\mathrm{IMCAO}$ ( $\mathrm{n}=8$ for each group). Data are presented as the mean $\pm S D$. ${ }^{*} P<0.05, A L A /$ control vehicle group vs. the sham group; $+P<0.05$, the ALA group vs. the control vehicle group

(A)
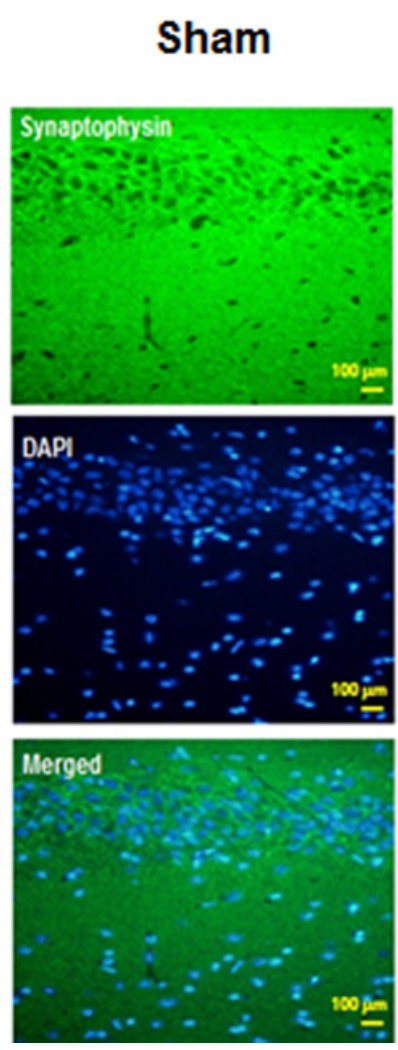

Synaptophysin stain

MCAO + Vehicle
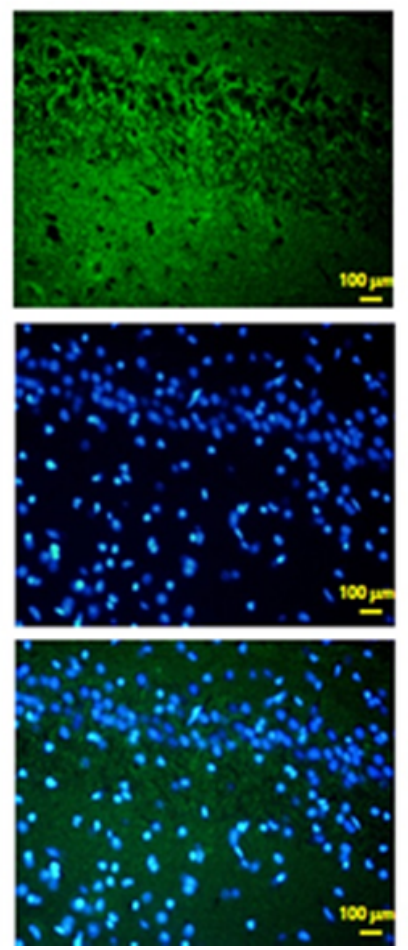

(B)

\section{MCAO + ALA}
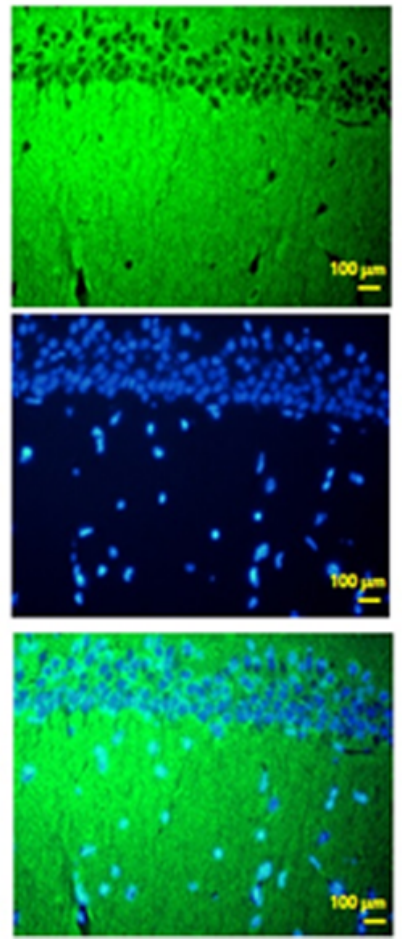


\section{Figure 4}

ALA preserved the integrity of neuronal synapses after cerebral I/R injury. (A) representative photographs of coronal hippocampal sections stained for synaptophysin and (B) quantification of the expression of synaptophysin in sham, and vehicle or ALA treated rats 7 days after $\mathrm{TMCAO}(\mathrm{n}=8$ for each group). Data are presented as the mean $\pm S D$. ${ }^{*} P<0.05, A L A /$ control vehicle group vs. the sham group; $+P<0.05$, the ALA group vs. the control vehicle group 